


WILEY-VCH

\title{
1,3-Dipolar cycloadditions of stabilized azomethine ylides and electrophilic alkenes mediated by a recyclable TSIL.AgOAC
} catalyst

\author{
David Lledó, ${ }^{[a]}$ Guillermo Grindlay, ${ }^{[a]}$ and José Miguel Sansano. ${ }^{[b]}$
}

\begin{abstract}
The synthesis of a task-specific ionic liquid (TSIL) based on a BMIM-ionic liquid phosphane is achieved by modification of the original procedure. It is used as ligand with $\mathrm{AgOAc}$, and the resulting complex is fully characterized. This catalytic system promotes the 1,3dipolar cycloaddition of stabilized azomethine ylides and electrophilic alkenes using a green methodology. The green charater is analized in an overall set of parameters finding the optimal conditions and individualized separation/purification methods for each cycloadduct. The determination of the most important green metrics is reported in this survey, demonstrating the value of this methodology in laboratory scale or even at an industrial level.
\end{abstract}

\section{Introduction}

The 1,3-dipolar cycloadditions (1,3-DCs) involving azomethine ylides and electron-poor alkenes are characterized by their total atom economy. ${ }^{[1,2]}$ In addition, high stereospecificity, regio-, and diastereoselectivity are very frequently achieved due the high control of the geometry of the dipole, as well as the preferential dipolarophile approach. These features convert this cycloaddition in a very attractive subject for implementing the green chemistry concepts. ${ }^{[3]}$

The 1,3-DCs performed with azomethine ylides generated via decarboxylative route of iminium salts have been very frequently associated to green processes. For example, heterogeneous graphene oxide, ${ }^{[4]}$ zinc oxide nanoparticles, ${ }^{[5]}$ heterogeneous silver nanoparticle ${ }^{[6]}$ bentonite ${ }^{[7]}$ were used as recyclable catalyst. The ability to run these reactions using multicomponent versions ${ }^{[8]}$ and the employment of more efficient microwave-assisted heating, ${ }^{[9,10]}$ were also adapted. Sequential thermal (MW) $[3+2]$ cycloaddition involving imino esters generated in situ was also

[a] Mr. D. Lledó and Prof. G. Grindlay.

University of Alicante, Department of Analytical Chemistry, Nutrition and Food Sciences, PO Box 99, 03080 Alicante, Spain.

https://cvnet.cpd.ua.es/curriculum-breve/es/lledo-garcia-david/32643 https://cvnet.cpd.ua.es/curriculum-breve/es/grindlay-lledoguillermo/9734

[b] Prof. J. M. Sansano.

University of Alicante, Department of Organic Chemistry, Centro de Innovación en Química Avanzada (ORFEO-CINQA) and Instituto de Síntesis Orgánica (ISO), PO Box 99, 03080 Alicante, Spain. https://cvnet.cpd.ua.es/curriculum-breve/es/sansano-gil-josemiguel $/ 10547$

Corresponding author: jmsansano@ua.es

Supporting information for this article is given via a link at the end of the document.((Please delete this text if not appropriate))

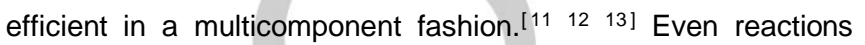
where products were isolated in good yield by simple filtration ${ }^{[14]}$ can be considered a good green approach. 2,2,2-Trifluoroethanol as a modern green solvent, ${ }^{[15]}$ PEG-400, ${ }^{[16]}$ ethyl lactate as a bio based green solvent, ${ }^{[17]}$ and ethanol, ${ }^{[18]}$ were also considered new green alternatives.

Concerning solvents, easily separable/extractable ionic liquids were also involved in 1,3-DC of azomethine ylides generated by decarboxylative route of iminium salts. The first [3+2] cycloaddition of azomethine ylides employing an ionic liquid medium allowed to prepare functionalized spiropyrrolidines. ${ }^{[19]}$ Other examples using 1-butyl-3-methylimidazolium tetrafluoroborate, [BMIM]BF ${ }_{4},{ }^{[20,21]}$ or $[\mathrm{BMIM}] \mathrm{Br}^{[22]}$ as ionic liquids, or ionic liquid-multicomponent-MW combinations, ${ }^{[23,24]}$ were described. In other contributions, the catalytic effect of the ionic liquid was demonstrated, due to the interaction of the heterocyclic hydrogen bonded at the 2-position of [BMIM]Br with the carbonyl group of the conjugated ketone. ${ }^{[25,26]}$ In the same line, spiro[pyrrolidine-benzofuran-3-one] were isolated in high yields (73-99\%) from the corresponding imino esters and cyclic unsaturated ketones using ionic liquids as catalysts. ${ }^{[27]}$

The employment of a chiral framework, containing an imidazolium-group-tagged planar chiral ferrocenyl oxazoline phosphane (FimiOAXP) ligand was designed. Here, the absence of an acidic hydrogen at the 2-position of the imidazolium ring avoided the formation of a copper(I) carbene, generating a conventional chiral ligand-copper(I) complex instead. In the presence of a weak base, the products were obtained, from iminoesters and several dipolarophiles, in satisfactory yields and with excellent enantioselectivity (up to $99 \%$ ee). ${ }^{[28]}$ These are examples of the so called task-specific ionic liquid (TSIL), which are designed and prepared for special reactions.

In this work, the synthesis and use of a (TSIL) BMIM-ionic liquidphosphane. $\mathrm{Ag}^{\prime}$, as recoverable catalyst, in the 1,3-DC of stabilized azomethine ylides and electrophilic alkenes is performed. We design an ambitious environmental friendly cycloaddition where, multicomponent process, room temperature (energetic efficiency), green solvent, inherent atom economy with no waste chemicals, high to excellent both diastereoselectivity and chemical yield, separation/isolation and purification of the cycloadduct in a simple and safer way, are the parameters to control.

\section{Results and Discussion}

In the enantioselective 1,3-DC of imino esters and maleimides, our group reported the application of a very stable (even to light 
exposure) chiral Binap. $\mathrm{AgClO}_{4}$, which was reused up to five catalytic cycles without apparent decrement of efficiency. ${ }^{[29]}$ Due to the interaction of the phosphino group with the silver cation and continuing with the development of new catalysts for this 1,3-DC, we envisaged ligand $1^{[30]}$ as a good candidate for this purpose. ${ }^{[31]}$ 1-Butyl-3-methylimidazolium hexafluorophosphate [BMIM]PF 6 was dissolved in extra-dry dichloromethane and treated with $n$ Buli at $-78{ }^{\circ} \mathrm{C}$ followed by the addition of chlorodiphenylphosphane giving final product 1 in $82 \%$ yield (Scheme 1).

$$
\underset{82 \%}{2 . \mathrm{Ph}_{2} \mathrm{PCl}}
$$

Scheme 1. Synthesis of ionic liquid ligand 1.

This ligand 1 was immediately combined with a silver salt and tested in the 1,3-DC between imino ester $\mathbf{2 a}$ and $N$ methylmaleimide (NMM) as benchmark reaction (Scheme 2, and Table 1). Too many parameters and reagents must be tested but we initially evaluated a reaction with $0.15 \mathrm{mmol}$ of both $2 \mathrm{a}$ and NMM in toluene ( $3 \mathrm{~mL}$ ) and triethylamine (1 equiv), together with a solution of $20 \mathrm{mg} \mathrm{BMIM}$, which contained AgOAc (1 mg, 0.006 $\mathrm{mmoL})$ and 1 (3.12mg, $0.007 \mathrm{mmoL})$. The mixture of BMIM, AgOAc and 1, was stirred for 1 hour previously, in the absence of light, before the addition of the other two components (Table 1). The employment of ethanol afforded higher yields than the reactions performed in dichloromethane, diethyl ether, THF, acetonitrile and isopropyl alcohol although all of them afforded similar diastereoselectivities (Table 1, entries 2-7). Finally, ethanol was selected because the reaction afforded a very clean ${ }^{1} \mathrm{H}$ NMR spectra and it is considered a valid green solvent. The presence of an added base as triethylamine was not necessary (Table 1, entry 8). The acetate anion could efficiently act as base and at this point, other basic silver salts as carbonate, benzoate or oxide were tested obtaining both lower chemical yields and diastereoselectivities of the final cycloadduct endo-3a (data not shown in Table 1). ${ }^{[32]}$ The most surprising result was obtained during the optimization of the amounts of catalyst, solvent, and time using the Plackett-Burman experimental design (see SI). ${ }^{[3]}$ When the reduction of the catalyst loading (1 mol\%) was done the reaction was completed in four hours almost quantitatively and with a 95:5 endo:exo ratio (Table 1, entry 9). The optimal amount of ethanol was $4 \mathrm{~mL}$ for the reaction of $0.2 \mathrm{mmol}$ of substrate $2 \mathrm{a}$ (Table 1, entries 9 and 10). Using the best conditions described before, the reaction was run in the absence of $\mathrm{AgOAc}$, that means, using both ionic liquids the process did not take place demonstrating the scarce catalytic activity of these species in this 1,3-DC between imino ester $\mathbf{2} \mathbf{a}$ and NMM (Table 1, entry 11 ).

For the purification of compound endo-3a, the mixture was refluxed at $78{ }^{\circ} \mathrm{C}$ for $30 \mathrm{~min}$ and the cycloadduct recrystallized at $4{ }^{\circ} \mathrm{C}$ taking advantage of the high solubility of these ionic liquids in ethanol after refluxing for 30 minutes. After this treatment, no epimerization of product endo-3a occurred. Next, this solution, containing BMIM, AgOAc and 1, was submitted to several identical processes, finding that the second process was completely analogous and reproducible (Table 1, entries 12 and 13). The third experiment with the same catalyst gave a lower, although important, yield ( $85 \%$ ) in very good $d r$, whilst the $4^{\text {th }}$ cycle was not so productive (Table 1, entries 14 and 15, respectively).

Table 1. Optimization of the 1,3-DC between $2 \mathrm{a}$ and NMM promoted by AgOAc. 1 complex.



[a] Isolated yield after the purification step. [b] Determined by ${ }^{1} \mathrm{H}$ NMR of the isolated compound. [c] Only $20 \mathrm{mg} \mathrm{BMIM}$ and $3.12 \mathrm{mg}$ of 1 were added.

The catalytic complex AgOAc. 1 was also characterized (Figure 1) obtaining the most representative data from the ${ }^{31} \mathrm{P}$ NMR experiments performed in deuterated chloroform. Meanwhile free phosphane 1 gave a signal at -26.5 ppm the silver complex spectra showed a peak at $22.6 \mathrm{ppm}$. The clear singlets observed in solution revealed that there is not the existence of different aggregates of the silver complex. In addition, the MS-ESI spectra also supported this fact because $\mathrm{m} / \mathrm{z}$ signals at 430 and 432 (cationic forms) corresponded to the existence of monomeric species (see SI). 


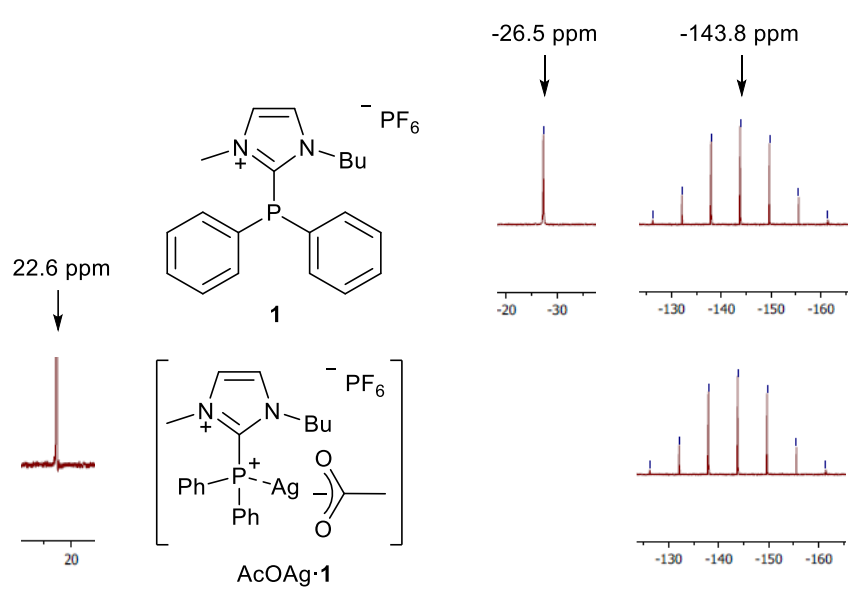

Figure 1. ${ }^{31} \mathrm{P}$ NMR shifts of ionic liquid 1 and complex 1.AgOAc.

The multicomponent version of this transformation was carried out using the same reaction conditions but with benzaldehyde and glycine methyl ester hydrochloride instead of the preformed imino ester $\mathbf{2} \mathbf{a}$. The generation of the free aminoester was attempted using trimethylamine in the presence of the catalysts and the dipolarophile, but the yield obtained of the cycloadduct endo-3a was very low $(<34 \%)$, even increasing the amount of complex $1 \cdot \mathrm{AgOAc}$ up to a $4 \mathrm{~mol} \%$. Besides, the reaction of imine $\mathbf{2 a}$ and NMM did not occurred neither in the absence of solvents nor in the presence of water.

Continuing with the reaction between several dipole precursors 2 and maleimides we found very interesting results. $\mathrm{N}$ Phenymaleimide reacted as NMM affording a lower yield (79\%) and similar diastereoselectivity (Table 2, compare entries 1 and 2). In both reactions, the purification of cycloadducts endo-3a and endo-3b was achieved by refluxing the mixture at $78^{\circ} \mathrm{C}$ for $30 \mathrm{~min}$ followed by recrystallization at $4{ }^{\circ} \mathrm{C}$. $\alpha$ - Substituted imino esters, derived from alanine $(\mathbf{2 b})$ and phenylalanine $(\mathbf{2 c})$, needed to react in a higher temperature (refluxing $\mathrm{EtOH}$ ) and using higher amount of catalyst to yield compounds endo-3c and endo-3d in 66 and $92 \%$ yield, respectively (Table 2 , entries 3 and 4 ). So, the reaction demonstrated to be sensitive to this substitution due to steric reasons. The diastereoselectivities were very high, especially in the case of the reaction with the phenylalanine derivative $(>99:<1)$. The purification of these two last heterocycles was accomplished by evaporating the $\mathrm{EtOH}$ followed by the addition of the minimum amount of $\mathrm{MeOH}$, which dissolved perfectly the ionic liquid and allowed the selective recrystallization of the corresponding cycloadducts at $-25^{\circ} \mathrm{C}$ (see, Table 2 ).

Table 2. Scope of the 1,3-DC between imino esters 2 and maleimides promoted by AgOAc. 1 complex.

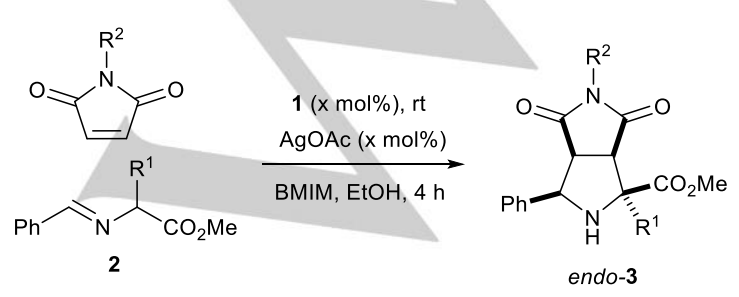

\begin{tabular}{llllllll}
\hline & $\begin{array}{l}\text { AgOAc.1 } \\
(\mathrm{mol} \%)\end{array}$ & $\mathrm{R}^{1}$ & $\mathrm{R}^{2}$ & $\begin{array}{l}\mathrm{T} \\
\left({ }^{\circ} \mathrm{C}\right)\end{array}$ & endo-3 & $\begin{array}{l}\text { Yield } \\
(\%)^{[\mathrm{a}]}\end{array}$ & endo/exo ${ }^{[\mathrm{b}]}$ \\
\hline 1 & 1 & $\mathrm{H}$ & $\mathrm{Me}$ & 25 & $\mathbf{3 a}^{[\mathrm{c}]}$ & 96 & $94: 6$ \\
2 & 1 & $\mathrm{H}$ & $\mathrm{Ph}$ & 25 & $\mathbf{3 b}^{[\mathrm{d}]}$ & 79 & $93: 7$ \\
3 & 4 & $\mathrm{Me}$ & $\mathrm{Me}$ & 78 & $\mathbf{3 c}^{[\mathrm{e}]}$ & 66 & $93: 7$ \\
4 & 4 & $\mathrm{Bn}$ & $\mathrm{Me}$ & 78 & $\mathbf{3}^{[\mathrm{e}]}$ & 92 & $>99:<1$
\end{tabular}

[a] Isolated yield after the purification step. [b] Determined by ${ }^{1} \mathrm{H}$ NMR of the isolated compound. [c] The reaction was completed in $4 \mathrm{~h}$. For the purification, the mixture was refluxed at $78{ }^{\circ} \mathrm{C}$ for $30 \mathrm{~min}$ and the cycloadduct recrystallized at $4^{\circ} \mathrm{C}$. [d] The reaction was completed in $17 \mathrm{~h}$. For the purification, the mixture was refluxed at $78^{\circ} \mathrm{C}$ for $30 \mathrm{~min}$ and the cycloadduct recrystallized at $4^{\circ} \mathrm{C}$. [e] The reaction was completed in $17 \mathrm{~h}$. For the purification, $\mathrm{EtOH}$ was evaporated and $\mathrm{MeOH}$ was added allowing the recrystallization at $-25^{\circ} \mathrm{C}$.

Other different representative dipolarophiles were assessed. For example, methyl acrylate reacted at room temperature (rt) in isopropyl alcohol (also considered as an appropriate green solvent) affording endo-3e in high yield and excellent diastereomeric ratio $(>99:<1$, Table 3 , entry 1$)$. The bulkier tertbutyl acrylate afforded a lower yield (76\%) and good diastereoselectivity $(92: 8)$ using the same reaction conditions (Table 3 , entry 2 ). The reaction with dimethyl fumatate required a higher temperature $\left(70{ }^{\circ} \mathrm{C}\right)$ to proceed $(68 \%$ isolated yield). This temperature was the cause of a decrement of the diastereoselection (Table 3, entry 3). Chalcone afforded major endo-3h cycloadduct in moderate isolated yield and with a notable diastereoselectivity (Table 3, entry 4). However, an opposite approach of the 1,2-bis(phenylsulfonyl)ethylene (BPSE) to the Wshape dipole took place, giving exclusively the exo-3j cycloadduct (see Figure 2) in both high yield and diastereoselectivity (Table 3 entry 5).

Another key detail of this full process involving 1,3-DC was the purification protocol (see Table 3). Each cycloadduct could be efficiently separated and purified using environmentally benign solvents and recrystallization tasks. In the most cases, the precipitation/crystallization of prolinates preferentially occurred and the washing step solubilized the remaining ionic liquid (Table 3 , entries 1, 2 and 4). However, in entry 3 of the Table 3, the complete BMIM precipitation took place at $-25^{\circ} \mathrm{C}$ obtaining pure compound endo-3g. The purification of the disulfone exo-3j was very difficult, but finally the solubility of the ionic liquid mixture in a 5:5:1 AcOEt:water:MeCN solution allowed to obtain pure cycloadduct (Table 3, entry 5).

Table 3. Scope of the 1,3-DC between imino ester $\mathbf{2 a}$ and other dipolarophiles promoted by AgOAc. 1 complex.






$\begin{array}{lllllll}3 & \mathrm{CO}_{2} \mathrm{Me} & \mathrm{CO}_{2} \mathrm{Me} & \begin{array}{l}\mathrm{EtOH} \\ 70^{\circ} \mathrm{C}\end{array} & \mathbf{3 g}^{[\mathrm{e}]} & 68 & 75: 25 \\ 4 & \mathrm{COPh} & \mathrm{Ph} & \mathrm{EtOH} & \mathbf{3 h}^{[\mathrm{f]}} & 45 & 89: 11 \\ 5 & \mathrm{SO}_{2} \mathrm{Ph} & \mathrm{SO}_{2} \mathrm{Ph} & \mathrm{EtOH} & \mathbf{3 j}^{[g]} & 89 & 9: 91\end{array}$

[a] Isolated yield after the purification step. [b] Determined by ${ }^{1} \mathrm{H}$ NMR of the isolated compound. [c] For the purification, the solvent was evaporated and the crude product was washed with $n-\mathrm{PrOH}$. [d] For the purification, the solvent was evaporated the reaction crude washed with tert-butyl methyl ether. [e] For the purification the crude mixture was stored at $-25^{\circ} \mathrm{C}$ overnight (BMIM precipitation occurred). [f] For the purification, the crude mixture was refluxed for $30 \mathrm{~min}$ and then recrystallization at $4{ }^{\circ} \mathrm{C}$ overnight. [g] For the purification, the solvent was evaporated, then AcOEt $(5 \mathrm{~mL})$ was added and the organic phase washed with water $(5 \mathrm{~mL})+\mathrm{CH}_{3} \mathrm{CN}(1 \mathrm{~mL})$.

The relative stereochemistry of all compounds was assigned by comparison of the ${ }^{1} \mathrm{H}$ NMR and ${ }^{13} \mathrm{C}$ NMR signals of the know compound described in the literature. ${ }^{[29]}$ This major endo-cycloadduct, obtained with a 2,5-cis-arrangement, is the result of the formation of the most stable $\mathrm{W}$-shape dipole whose geometry was controlled by this new ionic liquid phosphane 1.silver(I) complex. Then, the dipolarophile approached via an endo-fashion, that means, the electron-withdrawing group interacted with the metal center (Figure 2). However, stereoelectronic reasons can justify the opposite diastereoselection in the reaction with BPSE. This dipolarophile could interact with one of the phenyl groups bonded to the phosphorous atom, which facilitated the exo-approach (Figure 2). The hexafluorophosphate anion was not crucial in the explanation of this reverse diastereoselectivity because is placed in a long distance of the metal center.

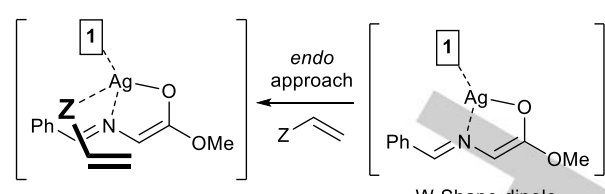

W-Shape dipole

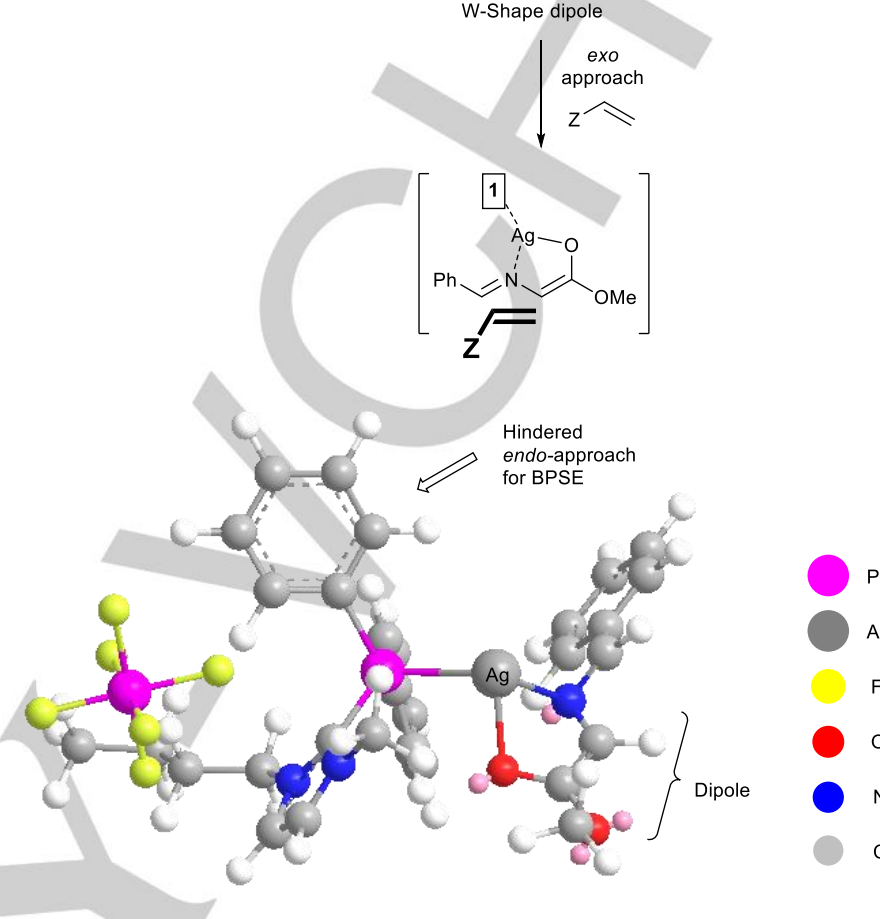

Figure 2. Endo- and exo-approaches onto the azomethine ylide with Wgeometry. Basic model of minimal energy justifying the exo-preference of BPSE.

For a comprehensive evaluation of the greenness and the eco-scale level of this 1,3-DC the reactions of the formation of endo-3a (A) and exo-3j (B) cycloadducts, following the Schemes of Tables 2 and 3, will be selected for the full analysis. Also, the whole process (C) to prepare endo-3a (supposing a 96\% yield) from glycine methyl ester hydrochloride, benzaldehyde and triethylamine will be assessed (Scheme 2). A comparison between $(\mathbf{A})$ and the analogous processes run in the presence of toluene (Tol) and dichloromethane (Dcm) will be also show in Table 4. Thus, green metrics calculations such as atom economy $(A E)$, atom efficiency (AEf), carbon efficiency $(C E)$, reaction mass efficiency (RME), optimum efficiency (OE), process mass intensity (PMI), E-factor (E), solvent intensity (SI) water intensity ${ }^{[34]}$ and eco-scale tool (E-S) (Table 4) $)^{[35]}$ were performed. The AE and CE were $100 \%$ except for method C due to the elimination of triethylammonium chloride as secondary product. RME and OE were identical to each other. RME, which is considered as useful metric to determine the greenness of the reaction considering reactant mass, atom economy and yield, was very high (96 and 89\%) for both A and B reactions, respectively. Clearly, Tol and Dcm methods are not as efficient and green reactions as A and B. A notable $62 \%$ of RME was determined for the whole process $\mathbf{C}$. Together with values of $\mathrm{OE}$, the higher the value of these five measurements is, the greener the process is (Table 4, columns 2-6). The last parameter (E-S) takes in consideration the quality (from the ecological and 
economical points of view) of the organic synthesis based on yield, safety, cost, general conditions, work-up and purification. The E$S$ value of process $\mathbf{A}$ is $85(0-100$ points range) which is considered an excellent economical/ecological procedure $(>75)$. In addition, procedure $\mathbf{B}$ has the penalty of using several solvents to perform the final liquid-liquid extraction and purification, meanwhile the whole reaction $\mathbf{C}$ has the penalty of toxicity of the commercially available basic components. Despite of these penalties, $\mathbf{B}$ and $\mathbf{C}$ got 71 and 73 marks, respectively, which are in the range of acceptable processes $(>50)$ but very close to the excellence limit (Table 4). E-S values of Tol and Dcm are notably less advantageous economical/ecological methods although both acceptable even $(>50)$.

$$
\underset{\cdot \mathrm{HCl}}{\mathrm{H}_{2} \mathrm{~N}} \widehat{\mathrm{CO}}_{2} \mathrm{Me}+\mathrm{Et}_{3} \mathrm{~N}+\mathrm{PhCHO} \longrightarrow \mathbf{2 a} \stackrel{\mathrm{NMM}}{\longrightarrow} \text { endo-3a }
$$

Scheme 2. Synthesis of endo-3a from commercially available precursors (process C).

Table 4. Green metrics (AE, AEF, CE, RME, OE, MP and E-S) for processes A, B and C.

\begin{tabular}{lllllll}
\hline Process & $\begin{array}{l}\text { AE } \\
(\%)\end{array}$ & $\begin{array}{l}\text { AEf } \\
(\%)\end{array}$ & $\begin{array}{l}\text { CE } \\
(\%)\end{array}$ & $\begin{array}{l}\text { RME } \\
(\%)\end{array}$ & $\begin{array}{l}\text { OE } \\
(\%)\end{array}$ & $\begin{array}{l}\text { E-S } \\
(0-100)\end{array}$ \\
\hline A & 100 & 96 & 100 & 96 & 96 & 85 \\
Tol & 100 & 88 & 100 & 79 & 88 & 58 \\
Dcm & 100 & 80 & 100 & 78 & 80 & 52 \\
B & 100 & 89 & 100 & 89 & 89 & 71 \\
C & 65 & 62 & 71 & 62 & 96 & 73
\end{tabular}

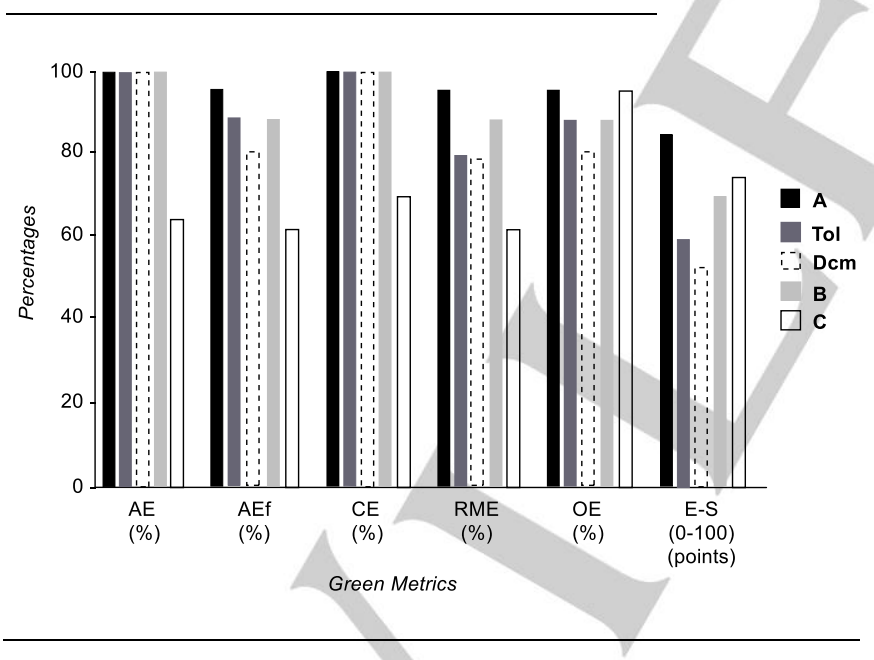

In the next PMI, E, SI and WI metrics, the lower values guarantee the better processes (Table 5). PMI and $E$ values are in the range of 52 and 66 in the preparation of endo-3a $(\mathbf{A})$ and exo-3j (B) cycloadducts. However, a much more interesting 1.04 $\mathrm{PMI}$ and $0.04 \mathrm{E}$ values could be obtained taking in account that the catalyst and the solvent were recycled in the preparation of endo-3a $\left(\mathbf{A}^{*}\right){ }^{34}$ Solvents constitute an important contribution in the solvent intensity (SI) parameter, thus the processes Dcm and
B had a very high metrics (85 and 151, respectively) (Table 5). WI index was 0 except for the example of exo-3j (B).

Table 5. Green metrics (PMI, E, SI and WI) for processes A and B.

\begin{tabular}{lllll}
\hline Process & PMI $(\mathrm{g} / \mathrm{g})$ & $\mathrm{E}(\mathrm{g} / \mathrm{g})$ & $\mathrm{SI}(\mathrm{g} / \mathrm{g})$ & WI $(\mathrm{g} / \mathrm{g})$ \\
\hline A & 52 & 51 & 51 & 0 \\
A $^{\star[a]}$ & 1.04 & 0.04 & 0 & 0 \\
Tol & 56 & 57 & 56 & 0 \\
Dcm & 87 & 86 & 85 & 0 \\
B & 66 & 65 & 151 & 58 \\
\hline
\end{tabular}

[a] The solvent and the catalyst were recovered and reused, so both of them were not included in the equation.

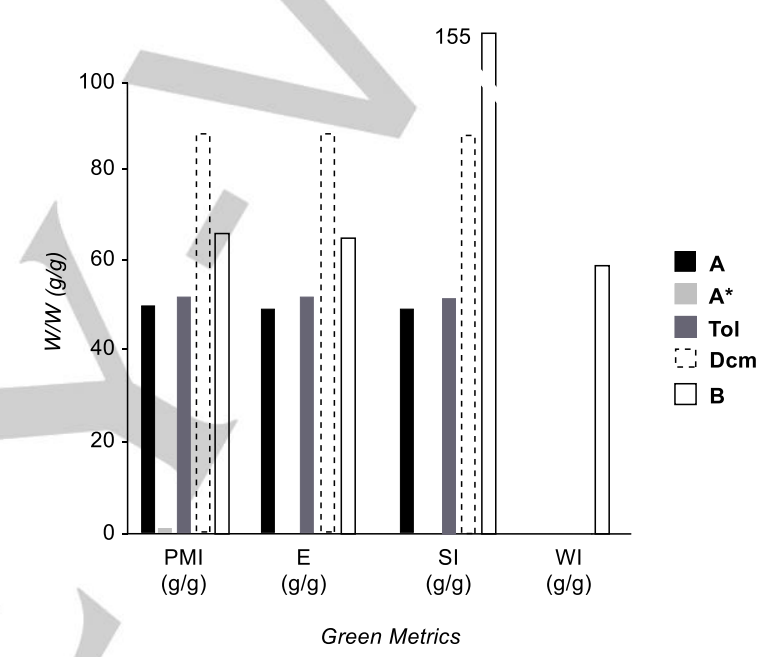

\section{Conclusions}

In this work, the preparation and use of an ionic liquid phosphane-silver acetate complex was efficiently focussed on the search of a green 1,3-DC between azomethine ylides and electrophilic alkenes. The green process was achieved using the minimal amount of an environmental benign solvent as ethanol (no solvent and water were not productive). The reaction was fast and completely reproducible. The separation of the catalytic complex plus solvent was demonstrated. The stability of the complex is good enough to recover it and reuse up to four batches. The small amount of the catalyst required and its easy synthesis contributed to increase the green character of this 1,3-DC. Another crucial point in a green process is the purification, and using this methodology it was possible to design an individual purification protocol for each compound. The metrics are excellent in the reaction involving NMM with methyl benzylideneaminoglycinate especially $\mathrm{AE}, \mathrm{CE}, \mathrm{RME}, \mathrm{PMI}, \mathrm{E}$ and the eco-scale parameter. 


\section{Experimental Section}

General 1,3-dipolar cycloaddition: To a solution of BMIM (20 mg) containing AgOAc (1 mg, $0.006 \mathrm{mmol}$ ) and the TSIL (3.12mg, $0.007 \mathrm{mmol})$ was allowed to stir for 1 hour in darkness to form the metallic complex. Then, the dipole and the dipolarophile were added together with the solvent and the mixture was stirred in darkness for the following 17 hours at a determined temperature. The purification steps are explained for each cycloadduct along the text.

See supporting information for additional details.

\section{Acknowledgments}

Keywords: Green chemistry - dipolar cycloaddition • azomethine ylides $\cdot$ TSIL $\cdot$ silver complex 
WILEY-VCH

Entry for the Table of Contents (Please choose one layout)

Layout 1:

\section{COMMUNICATION}

One of the best environmentally friendly 1,3-dipolar cycloadditions between imino esters and electrophilic alkenes was run using a versatile, efficient, stable and fully recoverable TSIL.AgOAc catalyst. Green metrics are excellent.

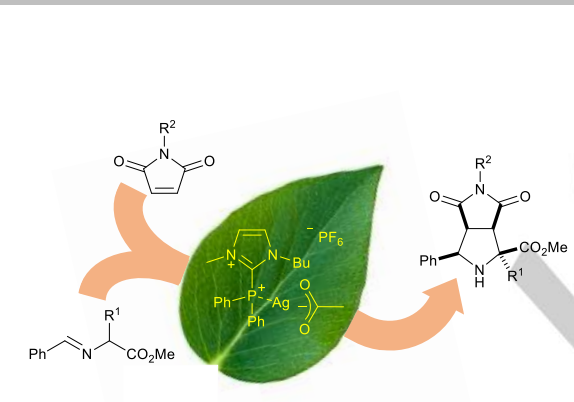

Green 1,3-dipolar cycloaddition

David Lledó, Guillermo Grindlay, José Miguel Sansano *

Page No. - Page No.

1,3-Dipolar cycloadditions of stabilized azomethine ylides and electrophilic alkenes mediated by a recyclable TSIL.AgOAc catalyst

\section{Notes and references}

[1] N. Arumugam, R. S. Kumar, A. I. Almansour, S. Perumal, Curr. Org. Chem. 2013, 17, 1929-1956.

[2] H. A. Döndas, M. de G. Retamosa, J. M. Sansano, Synthesis 2017, 49, 2819-2851.

[3] A. Ivanković, A. Dronjić, A. M. Bevanda, S. Talić, Intern. J. Sustain. Green Energy 2017, 6, 39-48.

[4] M. S. Reddy, N. S. Kumar, L. R. Chowhan, RSC Adv. 2018, 8, 35587-35593.

[5] N. Satish-Kumar, M. S. Reddy, V. R. Bheeram, S. B. Mukkamala, L. Raju-Chowhan, L. Chandrasekhara-Rao, Environ. Chem. Lett. 2018, 17, 455-464.

[6] S. Madhavan, S. Okamoto, ChemCatChem 2018, 10, 2014-2018.

[7] G. Sridhar, T. Gunasundari, R. Raghunathan, Tetrahedron Lett. 2007, 48, 319-322.

[8] J. Gonzales, N. V. S. D. K. Bhupathiraju, D. Hart, M. Yuen, M. P. Sifuentes, B. Samarxhiu, M. Maranan, N. Berisha, J. Batteas, C.-M. Drain, J. Org. Chem. 2018, 83, 6307-6314.

[9] B. Kalluraya, S. Mallya, K. A. Kumar J. Heterocycl. Chem. 2018, 55, 2075-2081.

[10] H. Peng, Z. Yang, S. Huang, T. Liu, Chin. J. Chem. 2010, 28, 1223-1228.

[11] A. Muthengi, X. Zhang, G. Dhawan, W. Zhang, F. Corsini, W. Zhang, Green Chem. 2018, 20, 3134-3139.

[12] A. Almansour, N. Arumugam, R. S. Kumar, G. Periyasami, H. A. Ghabbour, H.-K. Fun, Molecules 2015, $20,780-791$.

[13] M. Li, F.-M. Gong, L.-R. Wen, Z.-R. Li, Zhao-Rui, Eur. J. Org. Chem. 2011, 3482-3490.

[14] S. Nayak, S. K. Mishra, S. Bhakta, P. Panda, N. Baral, S. Mohapatra, C. S. Purohit, S. Chandra, P. Satha, Lett. Org. Chem. 2016, 13, 11-21.

[15] A. Dandia, R. Singh, S. Khan, S. Kumari, P. Soni, Tetrahedron Lett. 2015, 56, 4438-4444.

[16] J. Sindhu, H. Singh, J. M. Khurana, Mol. Divers. 2014, 18, 345-355.

[17] A. Dandia, A. K. Jain, A. K. Laxkar, Tetrahedron Lett. 2013, 54, 3929-3932.

[18] M. J. Taghizadeh, H. Arvinnezhad, S. Samadi, K. Jadidi, A. Javidan, B. Notash, Tetrahedron Lett. 2012, 53, 5148-5150.

[19] R. S. Kumar, A. I. Almansour, N. Arumugam, A. Basiri, Y. Kia, R. R. Kumar, Aust. J. Chem. 2015, 68(6), 863-871.

[20] A. Dandia, A. K. Jain, A. K. Laxkar, K. Ashok, D. S. Bhati, Tetrahedron 2013, 69, 2062-2069.

[21] R. Michael, S. Rajesh, B. D. Bala, S. Perumal, Tetrahedron Lett. 2012, 53, 5367-5371.

[22] R. Jain, K. Sharma, D. Kumar, Tetrahedron Lett. 2012, 53, 1993-1997.

[23] D. Gavaskar, A. R. S. Babu, R. Raghunathan M. Dharani, S. Balasubramanian, Steroids 2016, 109, 1-6.

[24] K. Jadidi, M. M. Moghaddam, K. Aghapoor, R. Gharemanzadeh, J. Chem. Res. 2007, 71-73.

[25] N. Arumugam, A. I. Almansour, R. S. Kumar, J. C. Menéndez, M. A. Sultan, U. Karama, H. A. Ghabbour, H.-K. Fun, Molecules 2015, 20, 16142-16153.

[26] D. Gavaskar, A. R. Suresh-Babu, R. Raghunathan, M. Dharani, S. Balasubramanian, J. Organomet. Chem. 2014, 768, 128-135.

[27] (a) C.-B. Zhang, P.-H. Dou, J. Zhang, Q.-Q. Wei, Y.-B. Wang, J.-Y. Zhu, J.-Y. Fu, T. Ding, ChemistrySelect 2016, 1, 4403-4407. (b) W.-Q. Hu, Y.-S. Cui, Z.-J. Wu, C.-B. Zhang, P.-H. Dou, S.-Y. Niu, J.-Y. Fu, Y. Liu, RSC Adv. 2015, 5, 70910-70914.

[28] L. Dai, D. Xu, X. Dong, Z. Zhou, Tetrahedron: Asymm. 2015, 26, 350-360. 


\section{WILEY-VCH}

[29] C. Nájera, M. de G. Retamosa, J. M. Sansano, Org. Lett. 2007, 9, 4025-4028.

[30] D. J. Brauer, K. W. Kottsieper, C. Liek, O. Stelzer, H. Waffenschmidt, P. Wasserscheid, J. Organomet. Chem. 2001, 630, 177-184.

[31] This ligand exhibited an extraordinary affinity by palladium(II) cation, see: (a) D. Lledó, G. Grindlay, J. Mora, L. Gras, J. M. Sansano, in preparation. (b) M. Gholinejad, F. Khosraviza, D. Lledó, G. Grindlay, C. Nájera, J. M. Sansano, J. Organomet. Chem. 2019, submitted.

[32] For silver carbonate and silver oxide, their higher basicity increased the deprotonation rate of the dipole and the diastereoselectivity could not be so highly controlled. In addition, the solubility of them could be the cause of the lower chemical yields achieved. Silver benzoate afforded slightly lower both chemical yields and $d r$ than the analogous transformation run with AgOAc.

[33] N. Sharif, Md. Z. Alam, Int. J. Engineer. Res. Technol. 2013, 2, 1-4.

[34] These metrics and their detailed calculations are fully and perfectly defined in ref. 11 .

[35] K. van Aken, L. Strekowski, L. Patiny, Beils. J. Org. Chem. 2006, 2, 1-7. 